\title{
Escalamiento de secciones a partir de módulos de elasticidad
}

\section{Scaling of sections from elasticity modules}

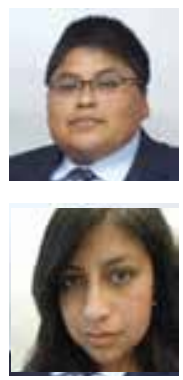

\section{Morales Luis}

Magister en Estructuras y Ciencias de los Materiales, docente titular Facultad de Ingeniería, Ciencias Físicas y Matemática de la UCE

Email: Iwmorales@uce.edu.ec

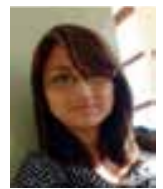

Coronel Armas María Gabriela

Estudiante de la Carrera de Ingeniería Civil, Facultad de Ingeniería, Ciencias Físicas y Matemática de la Universidad Central del Ecuador

Email: gabriela.coronel@outlook.com

\section{Resumen}

La modelación a escala, en acero, de un edificio de 9 pisos situado en la ciudad de Quito, sometido a cargas sísmicas en la mesa de vibración XY Shake Table III, de la Facultad de Ingeniería Ciencias Físicas y Matemática, a partir de la modelación matemática en ETABS, considera especificaciones establecidas por la Norma Ecuatoriana de la Construcción (NEC) para el diseño de los elementos estructurales y el análisis de los resultados, permitiendo determinar técnicas de construcción y tipo de acero a ser utilizados para la elaboración de modelos a escala que reflejen el comportamiento real de la edificación. Utilizando el proceso de investigación para el desarrollo del proyecto, se identifican los elementos estructurales de la edificación existente en planos arquitectónicos, para el desarrollo del pre diseño de los mismos y posteriormente su escalamiento a acero, utilizando módulos de elasticidad. Además, al ser sometidos a la misma carga sísmica, tanto el modelo matemático como el modelo a escala, se realiza la comparación de resultados para analizar el comportamiento estructural, con base en la normativa vigente. Para el uso de la mesa de vibración XY Shake Table III se realizará el procedimiento de escalamiento de sismos del ASCE/SEI 10, considerado por el Dr. Aguiar Falconí, R., \& García , H., utilizando eventos sísmicos registrados por el IG-EPN y sismos que podrían producirse en la ciudad de Quito, debido a las fallas geológicas presentes y a la energía acumulada en la corteza terrestre.

Palabras clave: modelación a escala; cargas sísmicas; mesa de vibración; modelación matemática; comportamiento estructural; escalamiento; norma ecuatoriana de la construcción.

\section{Abstract}

The modeling to steel-scale of a 9-floor building which is located in Quito city, under seismic loads on the XY vibration Table III table of the Engineering, Physical Sciences and Mathematics Faculty, based on mathematical modeling in ETABS, considers established specifications by the Ecuadorian Construction Standard (NEC) for the design of structural elements and the analysis of results, getting the determination of construction techniques and steel type to be used for the elaboration of scale models that reflect the real behavior of the building. Using the research process for the development of the project, the structural elements of the existing building are identified in architectural plans, for the development of the pre-design of the same and later its scaling to steel using elasticity modules. In addition, when subjected to the same seismic load, both the mathematical model and the scale model, the results are compared to analyze the structural behavior based on current regulations. For the use of the XY Shake Table III vibration table, the earthquake escalation procedure of the ASCE / SEI 10 will be carried out, considered by Dr. Aguiar Falconi, R., \& García, H., using seismic events registered by the IG -EPN and earthquakes that could occur in the city of Quito due to the present geological faults and the energy accumulated in the earth crust.

Keywords: scale modeling; seismic loads; vibration table; mathematical modeling; structural behavior; scaling; Ecuadorian construction regulation. 


\section{Introducción}

Un sismo no daña las edificaciones, es la fuerza de inercia, generada a partir de la vibración de la masa del edificio, la que produce dichos daños; su forma, las dimensiones y la configuración determinan la fuerza de afectación y resistencia ante la acción de los sismos; además, el peso de la edificación es lo que produce el colapso; generalmente los edificios caen verticalmente, no a los lados, como consecuencia de que las fuerzas laterales tienden a doblar y quebrar las columnas y muros estructurales, y la acción de la gravedad sobre la estructura debilitada produce dicho colapso. (Belriq, 2017).

Debido al crecimiento demográfico de la ciudad de Quito se ha incrementado la construcción de viviendas con diferentes materiales como: adobe, ladrillo, bloques y principalmente hormigón armado en sitios inestables, como quebradas rellenas de material suelto y en laderas empinadas, gran parte de las construcciones antiguas y actuales son informales, es decir, que carecen del uso de la normativa vigente y de mano de obra calificada, siendo un problema por la disminución del desempeño estructural de las mismas, de tal manera que, sí ocurriera un sismo de gran magnitud, los daños serían considerables. (Naya, 2010).

De acuerdo a los registros obtenidos en el Instituto Geofísico de la Escuela Politécnica Nacional (IG-EPN), en el año de 1587, en el norte de la ciudad de Quito, se produjo un sismo de magnitud Mw de 6.4 (Mw es una escala universal de magnitud que relaciona la energía sísmica liberada por el terremoto con las características físicas de la falla geológica que lo produce), asociado al sistema de fallas geológicas (fallas ciegas); desde esa fecha no se han vuelto a registrar sismos con magnitudes mayores a 6 grados, sin embargo, la ciudad presenta una alta peligrosidad sísmica. Sismos, como el producido en 1868, cuando la población de la ciudad era de 45.000 habitantes que vivían en un área de 4 km2 (IG-EPN, 2003), afectó de manera significativa las construcciones la zona urbana, al igual que el sismo del 10 de agosto de 1990 con una magnitud Mw de 5.3.

Para el desarrollo de esta investigación se consideran los registros sísmicos del 12 de agosto, 16 de agosto y 26 de diciembre, todos del 2014, aplicando el procedimiento de escalamiento del ASCE/SEI 10 para obtener registros sísmicos compatible con el espectro de la NEC, utilizando los factores de sitio respectivos. Debido a que la aceleración es muy baja en el escalamiento de dichos sismos, se consideran sismos impulsivos que podrían producirse en la ciudad de Quito para observar el comportamiento de los modelos, matemático y a escala. (NEC, 2015)

Es así, que esta investigación se realiza para escalar estructuras reales utilizando módulos de elasticidad, someterlas a cargas sísmicas en una mesa de vibra- ción y analizar el comportamiento que esta presenta, llegando a obtener resultados muy cercanos a la realidad y así conocer el comportamiento que desarrolla la edificación ante la acción de cargas sísmicas.

Debido a que el comportamiento estructural de la edificación ante la acción de una fuerza sísmica es considerado tanto para edificaciones de hormigón armado como para edificaciones de estructura metálica, se debe cumplir con las especificaciones de la normativa vigente para el diseño de cada uno de los elementos estructurales que la conforman, por lo que es necesario determinar la zona sísmica en la que se va a implantar la edificación, siendo fundamental para el desarrollo del trabajo de titulación basado en el análisis estructural del comportamiento de una edificación mediante el uso de un modelo matemáticos.

\section{Metodología}

La metodología del proyecto de investigación académica parte de la arquitectura de la edificación de 9 pisos, llamada Sauces Plaza 2, implantado en la ciudad de Quito; la observación permite ubicar los elementos estructurales horizontales y verticales como: vigas, losas y columnas respectivamente, que conforman la estructura, para realizar el pre diseño de los elementos estructurales antes mencionados, aplicando consultas bibliográficas en la normativa de construcción vigente y normativa internacional utilizada en el país.

Se obtienen las aceleraciones de los eventos sísmicos con mayor magnitud registrados por el IG-EPN en la ciudad de Quito desde el 2011, año en el que los acelerógrafos empezaron a registrar estas aceleraciones. Se calculan los espectros de diseño y se compara con el espectro de la NEC, se analiza los resultados de aceleraciones obtenidas y se observa que estas son muy bajas.

Estas aceleraciones de Quito, cargadas en la mesa de vibración no permiten apreciar el movimiento del modelo a escala, es así que, basándose en el estudio de "Los sismos impulsivos y la necesidad de considerar factores Near fault en el espectro para diseño de estructuras en Quito", (Aguiar Falconí \& Castillo, 2014), donde se afirma que Quito, al encontrarse en el sistema de fallas ciegas que atraviesa la ciudad, tiene gran probabilidad de ocurrencia de sismos impulsivos, se selecciona eventos impulsivos del PEER (Pacific Earthquake Engineering Research Center) en función del índice de impulsividad, que pudieran producirse en la ciudad de Quito por las fallas ciegas y la acumulación de energía en la corteza terrestre, con los cuales se diseña la estructura para que cumpla con los parámetros de diseño sísmico, establecidos en la normativa vigente. 
A partir de las dimensiones de los elementos estructurales obtenidas del pre diseño, se realiza la modelación matemática de la edificación en un software para análisis y diseño estructural que permite conocer el comportamiento de la estructura al ser sometida a una carga sísmica. Se realiza el análisis paso a paso, en el tiempo establecido en la Norma Ecuatoriana de Construcción, en su capítulo de Peligro Sísmico, considerando los factores de sitio. Para el ingreso de datos en la mesa de vibración XY Shake Table III, se realiza el escalamiento de sismos tomando en cuenta el procedimiento según el Dr. Aguiar y García (2014) que afirma la realización de escalamiento de sismos construyendo acelerogramas compatibles con un espectro.

Para la modelación a escala, se realiza el escalamiento de las secciones de los elementos estructurales de acuerdo al método de relación de módulos de elasticidad, este método fue seleccionado ya que se puede realizar un análisis de los materiales con los que se va a construir el modelo a escala y representar las propiedades de los materiales con los que se va a construir o está construida la edificación establecida para el análisis y a su vez se determina de manera rápida el comportamiento estructural que presenta cuando está sometida a cargas sísmicas, con la comparación de las aceleraciones tanto del modelo matemático como el modelo a escala. Además, se considera las dimensiones y la capacidad de la mesa de vibración donde se va a ejecutar el ensayo.

Como referencia del proyecto "Reforzamiento sísmico de paraboloides hiperbólicos aplicando en NEC-11" (Arciniega y Fuentes, 2012), se considera la relación de módulos de elasticidad para el escalamiento de las

secciones de los elementos estructurales, detallado a continuación:

\section{Datos:}

Esfuerzo máximo de compresión del hormigón.

$$
f^{\prime} \mathrm{c}=280 \mathrm{~kg} / \mathrm{cm}^{2}
$$

Límite de fluencia del acero.

$$
f y=4200 \mathrm{~kg} / \mathrm{cm}^{2}
$$

Módulo de elasticidad del hormigón.

$$
\begin{gathered}
E c=4.7 * \sqrt{28} \mathrm{GPa}=24.870 \mathrm{GPa} . \\
E c=253517.45 \mathrm{~kg} / \mathrm{cm}^{2}
\end{gathered}
$$

Módulo de elasticidad del acero.

$$
E s=2038736 \mathrm{~kg} / \mathrm{cm}^{2}
$$

Peso específico del hormigón.

$\gamma_{h}=2.4 t / m^{3}$
Relación de módulos de elasticidad.

$$
\begin{aligned}
& n=\frac{E c}{E s} \\
& n=\frac{253517.45 \frac{\mathrm{kg}}{\mathrm{cm}^{2}}}{\frac{2100000 \mathrm{~kg}}{\mathrm{~cm}^{2}}} E c . \text { (1) } \\
& n=0.121
\end{aligned}
$$

Se realiza el escalamiento de los elementos estructurales considerando la relación de módulos de elasticidad, a continuación, se presenta el procedimiento.

\section{Vigas:}

Se tiene la siguiente dimensión para todas las vigas de la estructura:

Base $(b)=45 \mathrm{~cm}$

Altura $(h)=60 \mathrm{~cm}$

Cálculo de la inercia del elemento.

Inercia en el sentido $x$.

$$
\begin{aligned}
& I x x=\frac{b * h^{3}}{12} \text { Ec. (2) } \\
& I x x=\frac{45 \mathrm{~cm} *(60 \mathrm{~cm})^{3}}{12} \\
& I x x=810000.0 \mathrm{~cm}^{4}
\end{aligned}
$$

\section{Área del elemento considerando su inercia.}

$$
I=\frac{b * h^{3}}{12}
$$

Considerando una sección cuadrada, $b=h$.

$$
I=\frac{h^{4}}{12}
$$

Dónde:

$$
\begin{aligned}
& h=\sqrt[4]{12 * I} \\
& h=\sqrt[4]{12 * 810000.0 \mathrm{~cm}^{4}} \\
& h=b=55.84 \mathrm{~cm}
\end{aligned}
$$

Cálculo de área del elemento:

$$
\begin{aligned}
& A=b * h \text { Ec. (3) } \\
& A=55.84 \mathrm{~cm} * 55.84 \mathrm{~cm} \\
& A=3117.69 \mathrm{~cm}^{2}
\end{aligned}
$$

Área equivalente en acero.

Para el cálculo del área equivalente en acero de la sección, se realiza el producto del área del elemento por la relación de módulos de elasticidad.

$$
\begin{aligned}
& \text { Aea }=\text { Área del elemento } * n \text { Ec. (4) } \\
& \text { Aea }=3117.69 \mathrm{~cm}^{2} * 0.121 \\
& \text { Aea }=376.38 \mathrm{~cm}^{2}
\end{aligned}
$$


Sección necesaria del elemento estructural en acero escalado.

Tomando en cuenta que las dimensiones de la mesa de vibración XY Shake Table III son de $71.1 \mathrm{~cm} \times 71.1$ $\mathrm{cm}$, la estructura en acero escalada debe encajar en la mesa, de tal manera que no sobrepase los límites.

Conociendo que la estructura real tiene por lados: $25.65 \mathrm{~m}$ y $15.6 \mathrm{~m}$, se debe encontrar la escala que permita que el modelo escalado encaje en la mesa de vibración.

$$
\begin{aligned}
& E=\frac{\text { Plano }}{\text { Terreno }}=\frac{1}{x} \text { Ec. (5) } \\
& \frac{1}{x}=\frac{71.1 \mathrm{~cm}}{2565 \mathrm{~cm}} \\
& x=\frac{2565 \mathrm{~cm}}{71.1 \mathrm{~cm}} \\
& x=36 \cong 40
\end{aligned}
$$

Es así como se ha considerado una escala de 1:40. Se tienen las dimensiones escaladas de eje a eje.

Se calcula el área de acero escalada:

$$
\text { Aescalada }=\frac{376.38 \mathrm{~cm}^{2}}{40^{2}}=0.24 \mathrm{~cm}^{2}
$$

Con el cálculo anterior, se obtiene un área de $1.95 \mathrm{~cm} 2$ de viga en acero escalada. Considerando que la sección de la viga no es rectangular, se calcula una relación de sus lados.

Datos:

$$
\begin{aligned}
\mathrm{b}=45 \mathrm{~cm} \text { y } \mathrm{h}=60 \mathrm{~cm} & \\
\text { Relación de lados } & =\frac{h}{b} \\
\text { Relación de lados } & =1.33 \\
\frac{h}{b} & =1.33 \\
h & =1.33 b
\end{aligned}
$$

Ec. (6)

Se calcula los lados de la viga considerando la relación de lados y el área de acero calculada:

$$
\begin{gathered}
\text { Aescalada }=b * h \\
\text { Aescalada }=b * 1.33 b \\
\text { Aescalada }=1.33 b^{2} \\
b=\sqrt{\frac{\text { Aescalada }}{1.33}}=\sqrt{\frac{0.24 \mathrm{~cm}^{2}}{1.33}}=0.4 \mathrm{~cm}
\end{gathered}
$$

Es así como la sección de viga escalada en acero es: $b=0.4 \mathrm{~cm}$ y $h=0.6 \mathrm{~cm}$.

\section{Columnas:}

Para el escalamiento de las columnas se aplica el mismo procedimiento realizado en vigas.

Se tiene la siguiente dimensión de una columna:

Base $(b)=40 \mathrm{~cm}$

Altura $(h)=60 \mathrm{~cm}$

Cálculo de inercias.

Inercia en el sentido $\mathrm{x}$.

$$
\begin{aligned}
& I x x=\frac{b * h^{3}}{12} \\
& I x x=\frac{40 \mathrm{~cm} *(60 \mathrm{~cm})^{3}}{12} \\
& I x x=720000.0 \mathrm{~cm}^{4}
\end{aligned}
$$

Área del elemento considerando su inercia.

$$
I=\frac{b * h^{3}}{12}
$$

Considerando una sección cuadrada, $\mathrm{b}=\mathrm{h}$.

$$
I=\frac{h^{4}}{12}
$$

Donde:

$$
\begin{aligned}
h & =\sqrt[4]{12 * I} \\
h & =\sqrt[4]{12 * 720000.0 \mathrm{~cm}^{4}} \\
h & =b=54.22 \mathrm{~cm}
\end{aligned}
$$

Cálculo de área del elemento:

$$
\begin{aligned}
& A=b * h \\
& A=54.22 \mathrm{~cm} * 54.22 \mathrm{~cm} \\
& A=2939.81 \mathrm{~cm}^{2}
\end{aligned}
$$

\section{Área equivalente en acero.}

Para el cálculo del área equivalente en acero de la sección, se realiza el producto del área del elemento por la relación de módulos de elasticidad.

$$
\begin{aligned}
& \text { Aea }=\text { Área del elemento } * n \\
& \text { Aea }=2939.81 \mathrm{~cm}^{2} * 0.121 \\
& \text { Aea }=354.90 \mathrm{~cm}^{2}
\end{aligned}
$$

\section{Sección necesaria del elemento estructural en acero escalado.} Se calcula el área de acero escalada:

$$
\begin{aligned}
& \text { Aescalada }=\frac{354.85 \mathrm{~cm}^{2}}{40^{2}} \\
& \text { Aescalada }=0.22 \mathrm{~cm}^{2}
\end{aligned}
$$

Teniendo el cálculo anterior que $h=1.33 \mathrm{~b}$ se calcula el otro lado de la viga:

$$
\begin{aligned}
& h=1.33 * 0.4 \mathrm{~cm} \\
& \boldsymbol{h}=\mathbf{0 . 6} \mathbf{c m}
\end{aligned}
$$


Con el cálculo anterior, se obtiene un área de $0.22 \mathrm{~cm}^{2}$ de columna en acero escalada. Considerando que la sección de la columna no es rectangular, se calcula una relación de sus lados.

Datos:

Base $=40 \mathrm{~cm}$

Altura $=60 \mathrm{~cm}$

$$
\begin{aligned}
& \text { Relación declados }=\frac{\bar{b}}{\text { Relación de lados }=1.50} \\
& \text { Re }
\end{aligned}
$$

$$
\begin{aligned}
& \frac{h}{b}=1.50 \\
& h=1.50 b
\end{aligned}
$$

Se calcula los lados de la columna considerando la relación de lados y el área de acero calculada:

$$
\begin{aligned}
\text { Aescalada } & =b * h \\
\text { Aescalada } & =b * 1.50 b \\
\text { Aescalada } & =1.50 \mathrm{~b}^{2} \\
b=\sqrt{\frac{\text { Aescalada }}{1.50}} & =\sqrt{\frac{0.22 \mathrm{~cm}^{2}}{1.50}}=0.4 \mathrm{~cm}
\end{aligned}
$$

Teniendo el cálculo anterior que $h=1.50 \mathrm{~b}$ se calcula el otro lado de la viga:

$$
h=1.50 * 0.4 \mathrm{~cm}=\mathbf{0 . 6} \mathbf{c m}
$$

Es así como la sección de columna escalada en acero es:

$$
b=0.4 \mathrm{~cm} \text { y } h=\mathbf{0 . 6} \mathbf{c m} \text {. }
$$

Para la obtención de una sección circular del elemento escalado, se realiza la equivalencia entre inercias entre una sección rectangular y circular. Con este análisis se calculará el diámetro equivalente a partir de una sección rectangular.

Inercia sección rectangular:

$$
I_{\mathbf{n}}=\frac{b * h^{3}}{12}
$$

Inercia sección circular:

$$
I_{\circ}=\frac{\pi * d^{4}}{64}
$$

Igualación de inercias.

$$
\begin{aligned}
& I_{*}=I_{\circ} \\
& \frac{b * h^{3}}{12}=\frac{\pi * d^{4}}{64}
\end{aligned}
$$

Con el análisis presentado se inicia el cálculo considerando la sección de vigas donde: $b=0.4 \mathrm{~cm}$ y $h=$ $0.6 \mathrm{~cm}$.

$$
\begin{aligned}
\frac{b * h^{3}}{12}=\frac{\pi * d^{4}}{64} \\
\frac{(0.6 \mathrm{~cm}) *(0.4 \mathrm{~cm})^{3}}{12}=\frac{\pi * d^{4}}{64} \\
d=\sqrt[4]{\frac{64 *(0.6 \mathrm{~cm}) *(0.4 \mathrm{~cm})^{3}}{12 * \pi}} \\
d=0.51 \mathrm{~cm} \\
d=5.1 \mathrm{~mm} \cong 5 \mathrm{~mm}
\end{aligned}
$$

Se aplica el mismo procedimiento para las secciones de columnas; a continuación, se presenta una tabla resumen, donde se encuentra la sección real, la sección escalada rectangular y finalmente la sección escalada circular: La construcción de secciones en acero, con las dimensiones establecidas por este procedimiento, rigidiza los elementos que conformarán el modelo a escala, lo que evita ver el movimiento del modelo a pesar de que tiene 9 pisos durante el ensayo.

Se comprueba el tipo de acero que será utilizado para la conformación de columnas, vigas y losa; el tipo de acero utilizado deberá permitir un adecuado comportamiento de la estructura al ser ensayada en la mesa de vibración.

Con el ensayo del modelo a escala, en la mesa de vibración se determinará el tipo de acero a ser utilizado, las técnicas constructivas y se comparará con los resultados obtenidos en el modelo matemático realizado.

\section{Peso del modelo a escala:}

Utilizando la escala 1:40 establecida para determinar las dimensiones del modelo a escala se considera:

Datos:

Peso específico hormigón $(\gamma \mathrm{H})=2.4 \mathrm{t} / \mathrm{m}_{3}$

Peso específico del acero $(\gamma A)=7.85 \mathrm{t} / \mathrm{m}_{3}$

$n=\frac{\gamma_{H}}{\gamma_{A}} \quad$ Ec. (7)

$n=\frac{2.4 \mathrm{t} / \mathrm{m} 3}{7.85 \mathrm{t} / \mathrm{m} 3}=0.306$

Peso del edificio del modelo matemático $(\mathrm{P})=2902.446 \mathrm{t}$.

Se utiliza la siguiente ecuación para teóricamente para cambiar la sección de hormigón a acero:

$$
\begin{aligned}
P_{\text {TOTAL }} & =P * n \\
P_{\text {TOTAL }}=P * n & =2902.446 * 0.306 \\
& =888.14 t
\end{aligned}
$$

Para realizar el escalamiento se considera la escala 1:40, de manera que se asume un escalamiento de 40 en los tres sentidos de los cuerpos geométricos. 


$$
\begin{aligned}
& \text { Pesc }=\frac{P_{\text {TOTAL }}}{E^{3}} \quad \text { Ec. }(9) \\
& \text { Pesc }=\frac{888.148 t}{40^{3}}=0.01388 \mathrm{t}=13.88 \mathrm{~kg}
\end{aligned}
$$

De esta manera se considera que el peso del modelo a escala con los materiales considerados para el método que se utilice, tenga un valor aproximado a $13.88 \mathrm{~kg}$.

\section{Elaboración del modelo a escala}

Para la elaboración del modelo a escala se consideran los materiales a ser utilizados y la técnica constructiva. Debido a que el modelo a escala es en acero, la manera más efectiva de unir los elementos que lo conforman es a través de la suelda, considerando el método de rigidez por piso.

Este procedimiento es complejo debido a las dimensiones de los alambres y al espesor de las láminas de acero utilizadas para la elaboración del modelo a escala.

Para realizar el armado del modelo a escala se siguió el siguiente procedimiento:

- Con el procedimiento anterior de escalamiento de secciones se hace un plano del modelo que va a ser ensayado para determinar la cantidad necesaria de material que será utilizado en su elaboración.

- Se procede a cortar cada uno de los materiales utilizados para la elaboración del modelo a escala y se arma los pórticos con el alambre colocando las columnas principales de $3 \mathrm{~mm}$, desde la base hasta el último piso, uniéndolas mediante las vigas de $2 \mathrm{~mm}$, establecidas para ese lado de los pórticos. Una vez que se tienen armados los pórticos, en el sentido más largo, se procede a unir nuevamente con las vigas, los pórticos en el otro sentido, hasta formar el modelo, tomando en la manipulación de los materiales, para evitar las deformaciones, durante el proceso de elaboración.

- Cuando se realice completamente el armado de las columnas y vigas, mediante la soldadura, se procede a determinar el peso del modelo a escala que está siendo elaborado para tener registros, antes de la colocación de la base y las losas de tol, considerando diferentes tipos de balanzas para tener una mejor precisión.

- Con las dimensiones establecidas se corta el tol de manera precisa para formar las losas de acero y formar finalmente el modelo a escala.

Se colocan las placas de tol para completar la carga establecida en el escalamiento.

De esta manera, igual que los toles de la losa, la base del modelo a escala es empotrado para impedir el movimiento del edificio escalado, una vez que esté colocado en la mesa de vibración XY Shake Table III para el ensayo.
- Se pesa nuevamente la estructura para considerar el peso final del modelo con la base de tol.

- Para mostrar estética en el modelo después de haber sido soldado completamente, se pinta de color negro las columnas y las losas de color gris.

Ver figuras de la 1 a la 8 en anexos (formato digital). De manera que el modelo a escala tiene las siguientes dimensiones:

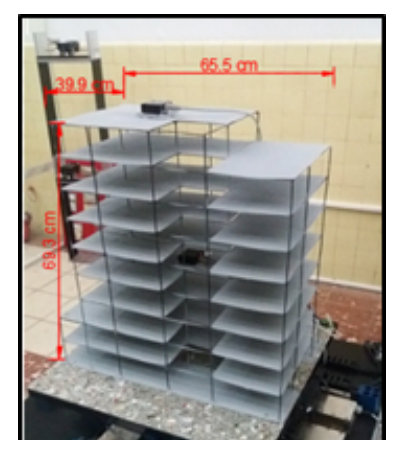

Figura 7: Modelo a escala con las dimensiones finales.

\section{Discusión de resultados}

De los ensayos realizados se obtienen los siguientes resultados del modelo matemático y del modelo escalado considerando la relación de los módulos de elasticidad de los materiales.

\section{Análisis tiempo historia de los sismos de Quito}

Para este análisis se comparan las gráficas "Tiempo historia" del modelo matemático y de las aceleraciones obtenidos por los acelerómetros en la mesa de vibración. Se analiza el modelo con la carga total, considerando la guía de experimentos a pequeña escala usando mesa de vibración y su comparación con predicciones analíticas. (UCIST, 2015). Para el ensayo se considera el número de piso en función del piso en el que se colocaron los acelerómetros, el piso 1 corresponde al piso 4 y el piso 2 se refiere al piso 9 en los modelos matemático y escala. De manera que se realiza el análisis de las aceleraciones cargadas en los modelos matemático y a escala, considerando la caracterización de registros acelerográficos obtenidos en el laboratorio. (Schmidt Díaz \& Quirós Serrano, 2007)

\section{Carga total.}

\section{Sismo del 12 de agosto del 2014.}

Se observa en la Fig. 10 (anexos), que la aceleración máxima es $0.21 \mathrm{~m} / \mathrm{s}^{2}$ que se encuentra de 3.62s. Mientras que en la Fig. 11 que corresponde a las aceleraciones de la mesa de vibración, la aceleración máxima promedio es de aproximadamente $0.027 \mathrm{~g}$, que corresponde a $0.27 \mathrm{~m} / \mathrm{s}^{2}$ en un tiempo de $3.57 \mathrm{~s}$ aproximadamente. La aceleración máxima de cada una de las gráficas tiene un tiempo similar, sin embargo, el valor de aceleración tiene una variación de $0.06 \mathrm{~m} / \mathrm{s}^{2}$.

En la Fig.12(anexos), la aceleración máxima es $0.22 \mathrm{~m} /$ $\mathrm{s}^{2}$ que se encuentra en un tiempo de 4.23s. Mientras 
que en la Fig. 13 que corresponde a las aceleraciones de la mesa de vibración, la aceleración máxima promedio es de aproximadamente $0.031 \mathrm{~g}$, que corresponde a $0.29 \mathrm{~m} / \mathrm{s}^{2}$ en un tiempo de $4.69 \mathrm{~s}$ aproximadamente. La aceleración máxima de cada una de las gráficas coincide en tiempo sin embargo el valor de aceleración es muy disperso teniendo una diferencia de $0.07 \mathrm{~m} / \mathrm{s}^{2}$.

\section{Sismo del 16 de agosto del 2014.}

Se observa en la Fig. 14 (anexos), que la aceleración máxima es $0.335 \mathrm{~m} / \mathrm{s}^{2}$ que se encuentra en un tiempo de 6.60s. Mientras que en la Fig. 15 que corresponde a las aceleraciones de la mesa de vibración, la aceleración máxima promedio es de aproximadamente $0.032 \mathrm{~g}$, que corresponde a $0.32 \mathrm{~m} / \mathrm{s}^{2}$ en un tiempo de $6.76 \mathrm{~s}$ aproximadamente. Se observa que la aceleración máxima de cada una de las gráficas tiene un tiempo similar, sin embargo, el valor de aceleración tiene una variación de $0.02 \mathrm{~m} / \mathrm{s}^{2}$.

Se observa en la Fig. 16 (anexos), que la aceleración máxima es $0.29 \mathrm{~m} / \mathrm{s}^{2}$ que se encuentra en un tiempo de 7.80s. Mientras que en la Fig. 17 que corresponde a las aceleraciones de la mesa de vibración, la aceleración máxima promedio es de aproximadamente $0.035 \mathrm{~g}$, que corresponde a $0.35 \mathrm{~m} / \mathrm{s}^{2}$ en un tiempo de 6.48s aproximadamente. La aceleración máxima de cada una de las gráficas se tiene un tiempo diferente con una variación de 1.32s, de la misma manera que el valor de aceleración que se encuentra muy disperso teniendo una diferencia de $0.06 \mathrm{~m} / \mathrm{s}^{2}$.

\section{Sismo del 26 de diciembre del 2014.}

Se observa en la Fig. 18 (anexos), que la aceleración máxima es $0.026 \mathrm{~m} / \mathrm{s}^{2}$ que se encuentra en un tiempo de 5.12s. Mientras que en la Fig. 19 que corresponde a las aceleraciones de la mesa de vibración, la aceleración máxima promedio es de aproximadamente $0.0025 \mathrm{~g}$, que corresponde a $0.025 \mathrm{~m} / \mathrm{s}^{2}$ en un tiempo de 5.62s aproximadamente. La aceleración máxima de cada una de las gráficas tiene un tiempo similar con variación de 50s, además, el valor de aceleración tiene una variación de $0.001 \mathrm{~m} / \mathrm{s}^{2}$.

En la Fig. 20, la aceleración máxima es $0.029 \mathrm{~m} / \mathrm{s}^{2}$ que se encuentra en un tiempo de 6.73s. Mientras que en la Fig. 21 que corresponde a las aceleraciones de la mesa de vibración, la aceleración máxima promedio es de aproximadamente $0.0026 \mathrm{~g}$, que corresponde a $0.026 \mathrm{~m} / \mathrm{s}^{2}$ en un tiempo de $6.48 \mathrm{~s}$ aproximadamente. Se observa que la aceleración máxima de cada una de las gráficas se tiene un tiempo diferente con una variación de 1.21s, de la misma manera que el valor de aceleración que se encuentra muy disperso teniendo una diferencia de $0.003 \mathrm{~m} / \mathrm{s}^{2}$.

\section{Análisis tiempo historia para sismos impulsivos.}

Para este análisis se comparan las gráficas "Time History" del modelo matemático y de las aceleraciones obtenidos por los acelerómetros en la mesa de vibración. Se analiza los casos de carga realizados: carga de vigas y columnas y carga adicional distribuida, ya que son los dos casos en los que mejor comportamiento tuvo el modelo en el ensayo. Para el ensayo se considera el número de piso en función del piso en el que se colocaron los acelerómetros, el piso 1 corresponde al piso 4 y el piso 2 se refiere al piso 9 en los modelos matemático y escala.

\section{Evento sísmico cape mendocino.}

\section{Carga vigas y columnas.}

Se observa en la Fig. 22, que la aceleración máxima es $24.15 \mathrm{~m} / \mathrm{s}^{2}$ que se encuentra en un tiempo de 3.3s. Mientras que en la Fig. 23 que corresponde a las aceleraciones de la mesa de vibración, la aceleración máxima promedio es de aproximadamente $2.9 \mathrm{~g}$, que corresponde a $29.60 \mathrm{~m} / \mathrm{s}^{2}$ en un tiempo de 3.30s aproximadamente. La aceleración máxima de cada una de las gráficas coincide en tiempo sin embargo el valor de aceleración es muy variado teniendo una diferencia de $5.45 \mathrm{~m} / \mathrm{s}^{2}$.

Se observa en la Fig. 24, que la aceleración máxima es $40.82 \mathrm{~m} / \mathrm{s}^{2}$ que se encuentra en un tiempo de 3.18s. Mientras que en la Fig. 25 que corresponde a las aceleraciones de la mesa de vibración, la aceleración máxima promedio es de aproximadamente $0.5 \mathrm{~g}$, que corresponde a $49.10 \mathrm{~m} / \mathrm{s}^{2}$ en un tiempo de $3 s$ aproximadamente. La aceleración máxima de cada una de las gráficas coincide en tiempo con variación mínima, sin embargo, el valor de aceleración es muy disperso teniendo una diferencia de $8.28 \mathrm{~m} / \mathrm{s}^{2}$.

Se observa en la Fig. 26, que la aceleración máxima es $7.75 \mathrm{~m} / \mathrm{s}^{2}$ que se encuentra en un tiempo de 3.3s. Mientras que en la Fig. 27 que corresponde a las aceleraciones de la mesa de vibración, la aceleración máxima promedio es de aproximadamente $0.41 \mathrm{~g}$, que corresponde a $4.12 \mathrm{~m} / \mathrm{s}^{2}$ en un tiempo de $3 \mathrm{~s}$ aproximadamente. La aceleración máxima de cada una de las gráficas coincide en tiempo con una variación mínima, sin embargo, el valor de aceleración presenta una diferencia de $3.63 \mathrm{~m} / \mathrm{s}^{2}$.

Se observa en la Fig. 28, que la aceleración máxima es $2.79 \mathrm{~m} / \mathrm{s}^{2}$ que se encuentra en un tiempo de $3.1 \mathrm{~s}$. Mientras que en la Fig. 29 que corresponde a las aceleraciones de la mesa de vibración, la aceleración máxima promedio es de aproximadamente $0.42 \mathrm{~g}$, que corresponde a $4.21 \mathrm{~m} / \mathrm{s}^{2}$ en un tiempo de 3s aproximadamente. La aceleración máxima de cada una de las 
gráficas coincide en tiempo y el valor de aceleración es muy cercano presentando una variación de $1.42 \mathrm{~m} / \mathrm{s}^{2}$.

\section{Carga adicional distribuida.}

\section{(fig. 30-fig.53 ver en anexos digitales)}

Se observa en la Fig. 30, que la aceleración máxima es $19.9 \mathrm{~m} / \mathrm{s}^{2}$ que se encuentra en un tiempo de 3.1s. Mientras que en la Fig. 31 que corresponde a las aceleraciones de la mesa de vibración, la aceleración máxima promedio es de aproximadamente $2.1 \mathrm{~g}$, que corresponde a $22.10 \mathrm{~m} / \mathrm{s}^{2}$ en un tiempo de $3 \mathrm{~s}$ aproximadamente. La aceleración máxima de cada una de las gráficas coincide en tiempo y su valor de aceleración varía en $2.20 \mathrm{~m} / \mathrm{s}^{2}$.

Se obtiene en la Fig. 32, que la aceleración máxima es $25.17 \mathrm{~m} / \mathrm{s}^{2}$ que se encuentra en un tiempo de $3.24 \mathrm{~s}$. Mientras que en la Fig. 33 que corresponde a las aceleraciones de la mesa de vibración, la aceleración máxima promedio es de aproximadamente $3.5 \mathrm{~g}$, que corresponde a $34.53 \mathrm{~m} / \mathrm{s}^{2}$ en un tiempo de $3.5 \mathrm{~s}$ aproximadamente. La aceleración máxima y el tiempo de cada una de las gráficas corresponden a valores similares. Teniendo en la aceleración una diferencia de $9.36 \mathrm{~m} / \mathrm{s}^{2}$ en un tiempo, en cada caso, de alrededor de $0.26 \mathrm{~s}$.

Se obtiene en la Fig. 34, que la aceleración máxima es $7.51 \mathrm{~m} / \mathrm{s}^{2}$ que se encuentra en un tiempo de 2.94s. Mientras que en la Fig. 35 que corresponde a las aceleraciones de la mesa de vibración, la aceleración máxima promedio es de aproximadamente $0.8 \mathrm{~g}$, que corresponde a $8.23 \mathrm{~m} / \mathrm{s}^{2}$ en un tiempo de $2.5 \mathrm{~s}$ aproximadamente. La aceleración máxima de cada una de las gráficas se encuentra en un tiempo muy aproximado teniendo una variación de $0.44 \mathrm{~s}$ y su valor de aceleración varía en $0.72 \mathrm{~m} / \mathrm{s}^{2}$.

Se obtiene en la Fig. 36, que la aceleración máxima es $11.30 \mathrm{~m} / \mathrm{s}^{2}$ que se encuentra en un tiempo de 2.94s. Mientras que en la Fig. 37 que corresponde a las aceleraciones de la mesa de vibración, la aceleración máxima promedio es de aproximadamente $3.1 \mathrm{~g}$, que corresponde a $10.00 \mathrm{~m} / \mathrm{s}^{2}$ en un tiempo de 2.52s aproximadamente. La aceleración máxima de cada una de las gráficas se encuentra en un tiempo muy aproximado teniendo una variación de 0.42s y su valor de aceleración también muy cercano con una variación de $1.30 \mathrm{~m} / \mathrm{s}^{2}$.

\section{Evento sísmico northridge}

\section{Carga vigas y columnas.}

En la Fig. 38 del modelo matemático, la aceleración máxima es $18.96 \mathrm{~m} / \mathrm{s}^{2}$ que se encuentra en un tiempo de 3.1s. Mientras que en la Fig. 39 que corresponde a las aceleraciones del modelo a escala, la aceleración máxima promedio es de aproximadamente $1.6 \mathrm{~g}$, que corresponde a $16.23 \mathrm{~m} / \mathrm{s}^{2}$ en un tiempo de $2.56 \mathrm{~s}$ aproximadamente. En este análisis se observa que la aceleración máxima de cada una de las gráficas se encuentra en un tiempo muy aproximado teniendo una variación de 0.54s mientras que los valores de aceleración tienen una variación de $2.73 \mathrm{~m} / \mathrm{s}^{2}$

Se obtiene en la Fig. 40 del modelo matemático, que la aceleración máxima es $14.19 \mathrm{~m} / \mathrm{s}^{2}$ que se encuentra en un tiempo de 9.68s. Mientras que en la Fig. 41 que corresponde a las aceleraciones del modelo a escala, la aceleración máxima promedio es de aproximadamente $1.7 \mathrm{~g}$, que corresponde a $16.80 \mathrm{~m} / \mathrm{s}^{2}$ en un tiempo de 2.55s aproximadamente. Las aceleraciones máximas de cada una de las gráficas no son similares teniendo una variación de $2.70 \mathrm{~m} / \mathrm{s} 2$, lo propio sucede con el tiempo, existiendo entre los dos análisis una variación de tiempo de $7.03 \mathrm{~s}$.

Se obtiene en la Fig. 42 del modelo matemático, que la aceleración máxima es $0.67 \mathrm{~m} / \mathrm{s}^{2}$ que se encuentra en un tiempo de 6.86s. Mientras que en la Fig. 43 que corresponde a las aceleraciones del modelo a escala, la aceleración máxima promedio es de aproximadamente $0.17 \mathrm{~g}$, que corresponde a $1.68 \mathrm{~m} / \mathrm{s}^{2}$ en un tiempo de 2.60s aproximadamente. Las aceleraciones máximas de cada una de las gráficas son similares teniendo una variación de $1.01 \mathrm{~m} / \mathrm{s}^{2}$, lo propio sucede con el tiempo, existiendo entre los dos análisis una variación de tiempo de 4.26s.

Se obtiene en la Fig. 44 del modelo matemático, una aceleración máxima es $24.63 \mathrm{~m} / \mathrm{s}^{2}$ que se encuentra en un tiempo de 6.88s. Mientras que en la Fig. 45 que corresponde a las aceleraciones del modelo a escala, la aceleración máxima promedio es de aproximadamente $0.2 \mathrm{~g}$, que corresponde a $21.10 \mathrm{~m} / \mathrm{s}^{2}$ en un tiempo de 2.98s aproximadamente. Las aceleraciones máximas de cada una de las gráficas no son similares teniendo una variación de $3.53 \mathrm{~m} / \mathrm{s}^{2}$, lo propio sucede con el tiempo, existiendo entre los dos análisis una variación de tiempo de 3.9s.

\section{Carga adicional distribuida.}

\section{Northridge piso 1 sentido $x$}

Se obtiene en la Fig. 46 del modelo matemático, una aceleración máxima es $8.33 \mathrm{~m} / \mathrm{s}^{2}$ que se encuentra en un tiempo de $6.58 \mathrm{~s}$. Mientras que en la Fig. 47 que corresponde a las aceleraciones del modelo a escala, la aceleración máxima promedio es de aproximadamente $0.79 \mathrm{~g}$, que corresponde a $7.93 \mathrm{~m} / \mathrm{s}^{2}$ en un tiempo de 2.5 s aproximadamente. La aceleración máxima de las dos gráficas no coincide en tiempo, obteniendo una variación de 4.08s y su valor de aceleración se aproxi$\mathrm{ma}$, variando en $0.40 \mathrm{~m} / \mathrm{s}^{2}$. 


\section{Northridge piso 2 sentido $x$}

Se obtiene en la Fig. 48 del modelo matemático, una aceleración máxima es $8.93 \mathrm{~m} / \mathrm{s}^{2}$ que se encuentra en un tiempo de 6.36s. Mientras que en la Fig. 49 que corresponde a las aceleraciones del modelo a escala, la aceleración máxima promedio es de aproximadamente $0.85 \mathrm{~g}$, que corresponde a $8.51 \mathrm{~m} / \mathrm{s}^{2}$ en un tiempo de 2.5s aproximadamente. La aceleración máxima de las dos gráficas no coincide en tiempo teniendo una variación menor de 3.86s, lo propio sucede con las aceleraciones máximas, se presenta una variación de $0.42 \mathrm{~m} /$ $\mathrm{s}^{2}$.

\section{Northridge piso 1 sentido y}

Se obtiene en la Fig. 50 del modelo matemático, una aceleración máxima es $14.47 \mathrm{~m} / \mathrm{s}^{2}$ que se encuentra en un tiempo de 7.1s. Mientras que en la Fig. 51 que corresponde a las aceleraciones del modelo a escala, la aceleración máxima promedio es de aproximadamente $1.4 \mathrm{~g}$, que corresponde a $15.10 \mathrm{~m} / \mathrm{s}^{2}$ en un tiempo de 3.1s aproximadamente. Las aceleraciones máximas de las gráficas son muy similares, obteniendo una variación de $0.63 \mathrm{~m} / \mathrm{s}^{2}$, sin embargo, la variación de tiempo que se presenta en este análisis es de $4.0 \mathrm{~s}$.

\section{Northridge piso 2 sentido $y$}

Se obtiene en la Fig. 52 del modelo matemático, una aceleración máxima es $22.20 \mathrm{~m} / \mathrm{s}^{2}$ que se encuentra en un tiempo de 7.26s. Mientras que en la Fig. 53 que corresponde a las aceleraciones del modelo a escala, la aceleración máxima promedio es de aproximadamente $1.4 \mathrm{~g}$, que corresponde a $13.73 \mathrm{~m} / \mathrm{s}^{2}$ en un tiempo de 3.5s aproximadamente. Las aceleraciones máximas de las gráficas presentan una variación de $8.47 \mathrm{~m} / \mathrm{s}^{2}$, en cuanto al tiempo, la variación que se presenta en este análisis es de 3.76s.

\section{Análisis de resultados}

Se realiza el cálculo de coeficiente de variación en función del promedio y de la desviación estándar de las aceleraciones respuestas obtenidas, para la comparación de resultados con los obtenidos en el modelo matemático del edificio de 9 pisos. En las tablas 3, 4 y 5, se resume el cálculo del coeficiente de variación y se observa que gran parte de los datos obtenidos tiene coeficientes de variación menores al 20\%, como un promedio del coeficiente para las aceleraciones se tiene un valor del $14 \%$ y para el tiempo del $9 \%$, descartando los que tienen porcentajes de variación más altos.

Además, en el estudio Shonkwiler y Miller se establece como aceptables los porcentajes de error entre $26 \%$ y $44 \%$ en el manual de las mesas de vibración donde se desarrollan "Pequeños experimentos de mesa de vibración y comparación con las predicciones analíti- cas", con lo que se valida el análisis entre el modelo matemático y el modelo a escala, para verificar que el edificio estudiado tiene un adecuado comportamiento estructural cuando está sometido a cargas sísmicas en la mesa de vibración, cuando se realiza el escalamiento de las secciones en función de la relación de módulos de elasticidad.

\section{Observaciones}

Con los ensayos realizados del modelo a escala, considerando el punto de partida el modelo matemático para el escalamiento de los elementos estructurales utilizando la relación de los módulos de elasticidad y al estar sometidos los dos modelos a las mismas cargas sísmicas, se obtienen resultados de aceleraciones con variaciones mínimas, permitiéndonos verificar el comportamiento adecuado de edificación ubicada en la ciudad de Quito.

La variación de los resultados en algunos de los eventos sísmicos se debe a que el modelo de la edificación se comporta de manera diferente cuando está sometido a los diferentes eventos sismos, principalmente por la colocación de los acelerómetros con los que se obtienen las aceleraciones respuestas.

Además, una de las consideraciones principales que deben estudiarse en futuras investigaciones, en base al proyecto de investigación realizado es la influencia de los desplazamientos en los resultados obtenidos para el análisis del comportamiento estructural de la edificación, por lo que es necesario analizar el escalamiento de los mismos, ya que con la variación del tiempo se cambia la intensidad de los sismos y principalmente la duración de la fase intensa.

\section{Conclusiones}

Basándonos en la importancia de realizar el análisis estructural de edificaciones existentes, se planteó el método de escalamiento, considerando la relación de módulos de elasticidad para la elaboración del modelo a escala, mismo que tiene un adecuado comportamiento estructural al estar sometido a las mismas cargas sísmicas que el modelo matemático, el cual fue diseñado de manera sismo resistente, cumpliendo con las secciones mínimas establecidas, por la normativa vigente. Se obtuvo una variación mínima en el análisis de las aceleraciones respuestas, determinándose resultados aceptables para el análisis realizado.

Con los sismos de Quito, en el análisis estructural del modelo a escala, se obtuvo aceleraciones muy bajas por lo que, al realizar el modelo matemático, éste se comportó de manera ideal cumpliendo con el diseño sismo resistente y en el ensayo del modelo a escala no se pudo apreciar el movimiento, es así que se seleccio- 
nó sismos impulsivos que pudieran ocurrir en la ciudad de Quito para verificar el comportamiento del edificio durante el ensayo y se pueda observar el movimiento de la estructura.

Se elabora varios modelos a escala con las secciones determinadas, considerando el método de escalamiento con la relación de los módulos de elasticidad y se realizan los ensayos hasta que se tienen las secciones adecuadas para el diseño, considerando columnas de $3 \mathrm{~mm}$ y vigas de $2 \mathrm{~mm}$, que son menos rígidas y presentan un comportamiento adecuado del modelo a escala.

Durante el ensayo, al observar el movimiento del modelo a escala y el comportamiento del material con el que fue elaborado, se concluye que, debido a las varias repeticiones de los ensayos, la estructura presentó fallas en sus uniones.

El modelo a escala que mejor representa el comportamiento estructural de un edificio, de acuerdo a la manera en la que se colocó el peso, es el que tiene carga adicional distribuida ya que se tiene un espesor comercial del material y el modelo es más compacto; a diferencia del modelo sin losas en el que no se pudo visualizar el movimiento y en el modelo con la carga total, donde se observó una vibración excesiva independiente al movimiento de la mesa. Se debe realizar una modelación matemática donde la estructura cumpla con los requisitos del diseño sismo resistente, para que se pueda iniciar el proceso de escalamiento.

Después de realizar los ensayos, se observó que el modelo a escala de 9 pisos que tenía una carga total no presentaba un adecuado comportamiento, por lo que no se consideró para el análisis de los resultados.

De acuerdo al análisis realizado en el modelo de 9 pisos, sometido a cargas sísmicas de Northridge y Cape, se observa que presenta mejor comportamiento durante el ensayo, la estructura que tiene carga adicional distribuida a comparación del modelo formado de solo vigas y columnas, donde en un tiempo similar tiene las aceleraciones máximas, pero con una alta diferencia en su valor.

Se considera que la manera más adecuada de elaborar el modelo a escala es con alambre para vigas y columnas, mientras que una placa de tol para las losas con un espesor comercial, para que el modelo se comporte adecuadamente en el ensayo; unidas a través de suelda para tener una mejor fijación, a pesar de que se mantenga en algunos casos muy rígida la unión de estos elementos por las dimensiones pequeñas del modelo elaborado.

Se observa que el sismo que afecta mayormente a las estructuras es el de Northridge, de manera que se ob- serva los más altos coeficientes de variación en los resultados de sus ensayos, a pesar de que se mantengan en el rango de $26 \%$ a $44 \%$ todos los datos, de acuerdo con la guía de experimentos a pequeña escala usando mesa de vibración y su comparación con predicciones analíticas.

Para la ejecución del proyecto de investigación se consideraron parámetros que fueron el punto de partida para el escalamiento y el desarrollo del modelo a escala, mismos que fueron modificándose en el transcurso del trabajo y que permitieron la obtención de resultados para su análisis.

\section{Referencias bibliográficas}

Aguiar Falconí, R., \& Castillo, D. (2013). Los sismos impulsivos y la necesidad de considerar factores "Near Fault" en el espectro para diseño de estructuras en Quito. Quito: Escuela Politécnica del Ejército

Aguiar Falconí, R., \& García , H. (2014). Escalamiento de sismos. Quito: Universidad de las Fuerzas Armadas.

Arciniegas Moncayo, C. B., \& Fuentes Montenegro, J. J. (2012). Reforzamiento sísmico de estructuras con paraboloides hiperbólicas aplicando NEC-11. Quito: Escuela Politécnica del Ejército (ESPE).

Belriq, M. (2017). Comportamiento de los edificios ante un sismo. Cidbimena Desastres Hn 11.

IG-EPN, I. G. (13 de enero de 2003). Instituto Geofísico de la Escuela Politécnica Nacional. Obtenido de IG EPN: http://www.igepn.edu.ec

Lázaro, L. (2015). Ensayo a escala de edificio de dos alturas sometido a desplazamientos horizontales. Barcelona: Universidad Politécnica de Barcelona.

López, P. M. (2003). La Guía para el Análisis y Diseño Estructural de Edificios de Hormigón Armado Ambato: Universidad Técnica de Ambato.

Naya, A. (2010). El Riesgo sísmico en Quito: Análisis y Simulaciones. Quito: Ciencias y gestión del territorio. NEC, N. E. (2015). Peligro Sísmico. Quito: CÓDIGO NEC-SE-DS.

Schmidt Díaz, V., \& Quirós Serrano, C. (2007). Caracterización de registros acelerográficos obtenidos en el laboratorio de Ingeniería Sísmica de la Universidad de Costa Rica. San José: Universidad de Costa Rica.

UCIST, U. C. (2015). Experimentos a pequeña escala usando mesa de vibración y su comparación con predicciones analíticas. San José: Laboratorio Nacional de Materiales y Modelos Estructurales Universidad de Costa Rica. 